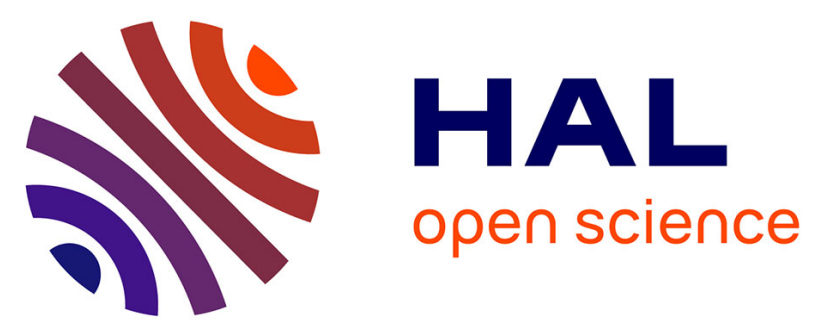

\title{
Nonlinear based Time Reversal acoustic applied to crack detection: Simulations and experiments
}

\author{
T. Goursolle, S. dos Santos, Olivier Bou Matar, S. Callé
}

\section{To cite this version:}

T. Goursolle, S. dos Santos, Olivier Bou Matar, S. Callé. Nonlinear based Time Reversal acoustic applied to crack detection: Simulations and experiments. International Journal of Non-Linear Mechanics, 2008, 43 (3), pp.170-177. 10.1016/j.ijnonlinmec.2007.12.008 . hal-00501768

\section{HAL Id: hal-00501768 \\ https://hal.science/hal-00501768}

Submitted on 12 Jul 2010

HAL is a multi-disciplinary open access archive for the deposit and dissemination of scientific research documents, whether they are published or not. The documents may come from teaching and research institutions in France or abroad, or from public or private research centers.
L'archive ouverte pluridisciplinaire HAL, est destinée au dépôt et à la diffusion de documents scientifiques de niveau recherche, publiés ou non, émanant des établissements d'enseignement et de recherche français ou étrangers, des laboratoires publics ou privés. 


\section{Author's Accepted Manuscript}

INTERNATIONAL JOURNAL OF

NON-LINEAR

MECHANICS

Nonlinear based Time Reversal acoustic applied to crack detection: Simulations and experiments

T. Goursolle, S. Dos Santos, O. Bou Matar, S. Callé

PII:

S0020-7462(07)00231-4

DOI:

doi:10.1016/j.ijnonlinmec.2007.12.008

Reference:

NLM 1425

To appear in: International Journal of NonLinear Mechanics

Received date: 23 May 2007

Revised date: 23 November 2007

Accepted date: 18 December 2007

Cite this article as: T. Goursolle, S. Dos Santos, O. Bou Matar and S. Callé, Nonlinear based Time Reversal acoustic applied to crack detection: Simulations and experiments, International Journal of Non-Linear Mechanics (2007), doi:10.1016/j.ijnonlinmec.2007.12.008

This is a PDF file of an unedited manuscript that has been accepted for publication. As a service to our customers we are providing this early version of the manuscript. The manuscript will undergo copyediting, typesetting, and review of the resulting galley proof before it is published in its final citable form. Please note that during the production process errors may be discovered which could affect the content, and all legal disclaimers that apply to the journal pertain. 


\title{
NonLinear based Time Reversal Acoustic applied to crack detection: Simulations and Experiments
}

\author{
T. Goursolle ${ }^{a}$, S. Dos Santos ${ }^{a}$, O. Bou Matar ${ }^{b}$ and S. Calléc \\ ${ }^{a}$ ENI Val de Loire, LUSSI de l'Université François Rabelais, FRE 2448 CNRS, \\ Rue de la Chocolaterie BP 3410, F-41034 Blois cedex, France \\ ${ }^{b}$ European Associated Laboratory in Nonlinear Magneto-Acoustics (LEMAC) / \\ IEMN-DOAE, UMR CNRS 8520, Ecole Centrale de Lille, F-59651 Villeneuve \\ d'Ascq, France \\ ${ }^{c}$ LUSSI de l'Université François Rabelais, FRE 2448 CNRS, \\ 10bis boulevard Tonnellé, F-37032 Tours Cedex1, France
}

\begin{abstract}
In nondestructive evaluation, Nonlinear Elastic Wave Spectroscopy (NEWS) methods represent powerful tools to explore damaged zones in a sample. The combination of these methods with Time Reversal (TR) process can be used to either increase the stress on the retrofocusing position or to retrofocuse elastic waves on the defect, when only the nonlinear components of the received signal are time reversed. In this paper, we propose two different approaches to detect cracks in metallic samples in coupling NEWS methods and TR process. The first one, NEWS-TR, is defined by sending back only the nonlinear components which are preliminary time-reversed. Two different techniques to filter nonlinear components have been numerically studied: classical harmonic filter and pulse inversion. In these two cases, performances of retrofocusing on different defect positions are analysed and compared. The second approach, TR-NEWS, consists in measuring the nonlinear signature on the focal spot. An experimental study of parametric interaction between two reversed signals $\left(f_{1}\right.$ and $\left.f_{2}\right)$ is led. Measurements of components at $f_{2}-f_{1}$ and $f_{2}+f_{1}$ around a crack are performed and discussed.
\end{abstract}

Key words: nonlinear acoustics, time reversal acoustics, nonclassical nonlinearity, harmonic generation, parametric interaction.

PACS: 43.25.Dc, 43.40.Le, 43.60.Tj

Email address: thomas.goursolle@univ-tours.fr. 


\section{Introduction}

In the last few years, a strong interest for the Nonlinear Elastic Wave Spectroscopy (NEWS) methods applied to nondestructive testing has grown [1]. Different studies of this behavior have been applied on a large range of materials : harmonic analysis [2], parametric interactions [3], modulation of amplitude [4] and phase [5] and studies of resonance frequency $[6,7]$.

It is established that cracked materials contain small soft features within a hard matrix producing a very large nonlinear response[8,9]. First observed in granular media [8], this response, which describes cracks in metallic samples too, corresponds to an nonlinear (called "nonclassical") effect characterized by an hysteretic behavior. Furthermore, Time Reversal (TR) $[10,11]$ process, which allows the focusing of signal on the source position, has been recently demonstrated to be highly useful for ultrasonic imaging in the nonlinear regime $[12,13,14]$. Several methodologies can be derived from Nonlinearity based Time Reversal concept depending on the fact that time reversal is used as a tool for energy focusing in order to generate nonlinear stress or used as a tool for nonlinear source identification (fig. 1).

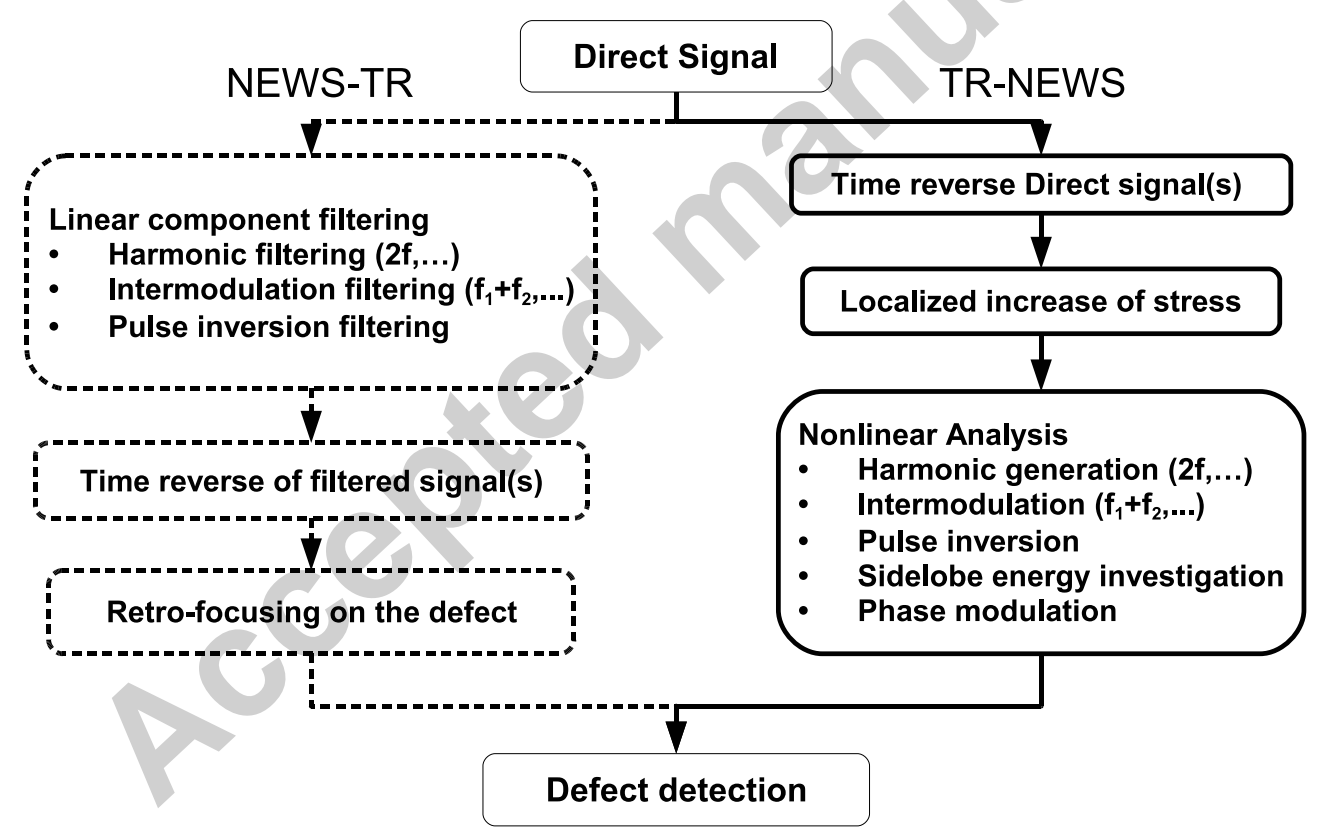

Fig. 1. Defect detection methods and nonlinear effects measured.

These two methodologies are defined TR-NEWS, with nonlinear analysis as a post-processing of time reversal, and NEWS-TR, with nonlinear analysis as a pre-processing of time reversal $[15,16]$. TR-NEWS method consists in locally increasing the stress field using properties of linear TR and subsequently applying a nonlinear analysis. This method has been experimentally demon- 
strated [12] and seems to have a wide potential for nondestructive application $[13,14]$. The NEWS-TR application is based on signal retrofocusing on the defect position when only the nonlinear components of the received signal are time reversed $[17,19,16]$. This method has only been validated experimentally recently [20].

A numerical study of NEWS-TR (left part of fig. 1) is investigated in section 2 thanks to a 2D pseudo spectral algorithm which takes into account the nonlinearity due to a defect zone producing a nonclassical nonlinearity linked to an hysteretic behavior. The nonlinear signature of harmonic generation is tested with two different filtering techniques : harmonic filtering and pulse inversion. In Section 3, a experimental TR-NEWS method combining TR and parametric interaction is developed. The TR process permits to increase the stress and to guarantee that both signals arrive at the same time at the same point. In order to investigate the nonlinear signature on the focus area, dependence of the parametric interaction components $f_{L}=f_{2}-f_{1}$ and $f_{H}=f_{2}+f_{1}$ versus amplitude of the fundamental components at the frequencies $f_{1}$ and $f_{2}$ is achieved.

\section{Numerical study of NEWS-TR}

\subsection{Numerical method description}

Considering the propagation of elastic waves in an heterogeneous solid medium where damaged zone is modelized with an hysteretic nonlinear behavior, the Newton's second law can be written:

$$
\frac{\partial v_{i}}{\partial t}=\frac{1}{\rho_{0}} \frac{\partial \tau_{i j}}{\partial x_{j}}
$$

where $x_{j}$ are the position vector components, $\rho_{0}$ is the volumetric mass density, $v_{i}$ are the particle velocity vector components and $\tau_{i j}$ are the stress tensor components. A 2D numerical method based on the pseudo-spectral algorithm [15] has been developed in order to solve this equation where nonclassical property is introduced with an hysteretic behavior.

\subsubsection{Hysteretic behavior modelisation}

The hysteretic nonlinear behavior, based on Preisach-Mayergoz (PM) model [21] and first introduced by McCall et al. [9], has been used. Elastic constants are calculated at each time step adding the contributions of $N_{0}=5$ hysteretic elementary units (HEU) to the deformation imposed by the elastic wave. Each 
HEU is described (fig. 2) by two characteristic stresses $\tau_{c}$ and $\tau_{o}$ which correspond to two states when the stress increases or decreases, respectively. One state is called "closed" state and the other one "open" state. For each cell of the calculation grid, representing mesoscopic description level of the medium, $N_{0} \mathrm{HEU}$ are considered with different values of the two characteristic stresses, depending on a distribution function $f\left(\tau_{c}, \tau_{o}\right)$ defined for the PM space [22]. Our implementation of the PM-space is based on the multiscale approach developed by Van Den Abeele et al. [23]. The elementary hysteretic elements, which have been considered here, is the one used by Scalenderi et al. [24] implemented in the LISA code, as shown on Fig.2.

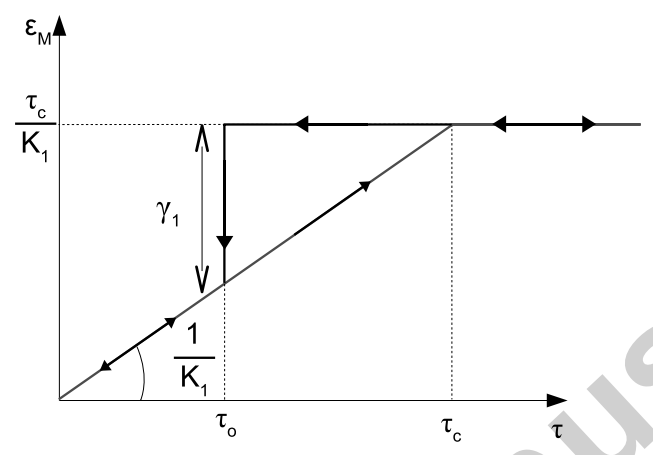

Fig. 2. Elementary hysteretic elements used in the computation of the PM-space model. In each case, one state corresponds to an open state and the other one to a closed state with $\tau_{o}$ and $\tau_{c}$ the associated transition stresses, respectively.

In order to extend this solver in two dimensions, Kelvin notation [25] has been used to modify elastic coefficient tensor. These components are calculated at each time step. Details of the calculation can be found in ref [16].

\subsubsection{Pseudo-spectral model}

The numerical technique used to solve the system of differential equations presented in the preceding part is a pseudo-spectral wave solver developed in our laboratory [26]. This method consists in computing spatial derivatives in Fourier domain by means of a fast Fourier transform(FFT) [27]:

$$
\frac{\partial f(x)}{\partial x}=F F T^{-1}\left[\left(j k_{x} e^{ \pm j k_{x} \frac{\Delta x}{2}}\right) \cdot F F T[f(x)]\right],
$$

where $F F T^{-1}$ is the inverse Fast Fourier Transform, and $k_{x}$ and $\Delta x$ are the wave number and the spatial step of the numerical grid in the $x$ direction, respectively. This algorithm has a better numerical stability and does not require a large number of nodes per wavelength compared to other numerical algorithms $[28,29]$. This advantage is of great importance in such a nonlinear study 
where several harmonics must be taken into account. To reduce numerical artifacts in simulations for a heterogeneous medium, a staggered spatial grid implementation is used. Moreover, a staggered fourth-order Adams-Bashforth method is used to update stress and particle velocity at alternating half time steps [30].

\subsection{NEWS-TR simulations}

\subsubsection{Simulation parameters}

The NEWS-TR method (left part on fig.1) investigated in these simulations consists in rebroadcasting the nonlinear signature of received signal alone and in examining the focused signal on the nonlinearity source. Two filtering methods, i.e. harmonic filtering and pulse inversion, have been studied to extract the nonlinear signature.

Geometry and some material characteristics of the 2D specimen $(300 \mathrm{~mm} \times$ $500 \mathrm{~mm}$ aluminum sample) are shown in Fig. 3. A $10 \mathrm{~mm} \times 50 \mathrm{~mm}$-source emits the elastic wave in the bulk with the following spatio-temporal form:

$$
f(x, z, t)= \pm p_{0} \cdot \sin \left(2 \pi f_{c} t\right) \cdot e^{-\frac{1}{2}\left(\frac{t}{w}\right)^{2}}\left(1-e^{-\left(\frac{t}{s_{w}}\right)^{2}}\right) \cdot e^{\frac{1}{2}\left(\frac{x-x_{s}}{w_{x}}\right)^{2}} \cdot e^{\frac{1}{2}\left(\frac{z-z_{s}}{w_{z}}\right)^{2}}
$$

where $f_{c}=200 \mathrm{kHz}$ is the source frequency, $p_{0}=50 \mathrm{kPa}$ is the initial pressure and $w=\frac{2}{f_{c}}, s_{w}=\frac{3}{f_{c}}, w_{x}=0.15, w_{z}=0.75, x_{s}=12,5 \mathrm{~cm}$ and $z_{s}=15 \mathrm{~cm}$ are emission parameters.

The receivers are composed of four lines (1D array of 112 elements) and simulations with two different length receivers $(300 \mathrm{~mm}$ and $165 \mathrm{~mm}$ ) have been done. The damaged zone (with an area of $56 \mathrm{~mm}^{2}$ ), associated to the defect position, presents an hysteretic nonlinear behavior. Acoustical properties of the rest of sample are linear. Consequently, the nonlinear signature is only present on the defect. Two different defect positions are chosen to study their influence on the retrofocusing quality (Fig. 3). 


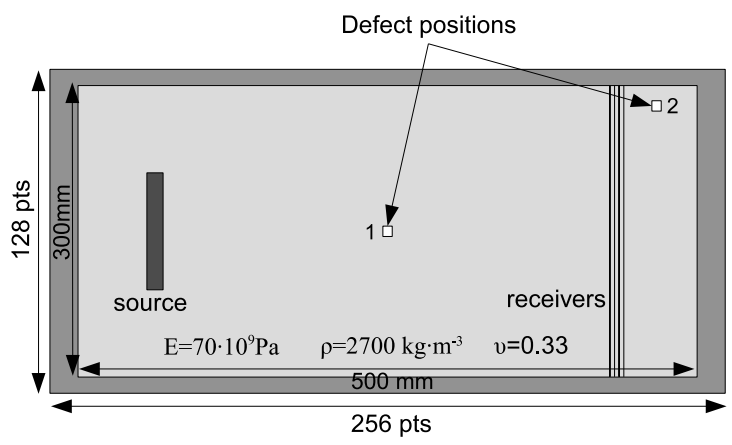

Fig. 3. Geometry of simulation domain with source, receivers, defect positions and material characteristics.

\subsubsection{Filtering methods}

In order to rebroadcast in the sample only the nonlinear parts (harmonics) of the received signal, two filtering methods were investigated. The first one consists in selecting only the nonlinear signature contained in the response signals and rebroadcasting merely this part into the medium by the time reverse mirror. Third harmonic is mainly extracted from received signal by means of a classical fourth order Butterworth filter.

An alternative filtering procedure, called Pulse Inversion (PI) filtering [31], is based on the fact that the phase inversion of a pulse excitation signal will lead to the exact phase inverted response signal within a linear medium. In a nonlinear (or microdamaged) material, this is not the case due to the generation of harmonics. Advantage of this observation is taken by adding the responses from two phase-inverted pulses (positive and negative) and sending back the sum at the receivers which is essentially composed of even harmonic components. Doing so, only the relevant information on the local nonlinearities is reversed and sent back into the material.

\subsubsection{NEWS-TR with harmonic generation}

In order to localize the damaged zone on the bulk, the direct signal, which has been propagated in the sample and received by the four lines, is filtered using one of the two filtering methods previously described in order to keep only the nonlinear components. Visualization of the spatial and temporal retrofocusing can be done by extracting the maximum of the time evolution of the stress for each position in the sample. Figure 4 illustrates the maximum amplitude of stress (along $y$ axis) for the defect in the center (position 1) and the defect in the corner (position 2). Figures 4(a) and 4(b) represent the maximum amplitude plots in the case of a central defect (position 1) with harmonic filtering and PI methods, respectively. In the same way, Figures 4(c) and 4(d) represent the maximum amplitude plots in the case of a defect near the edge (position 2) with these two filtering methods. 


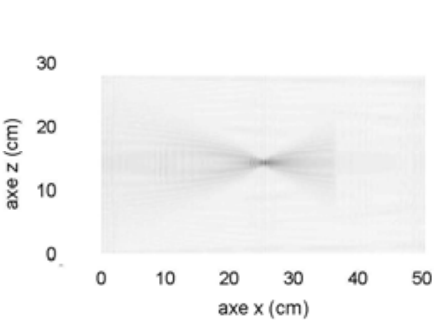

(a)

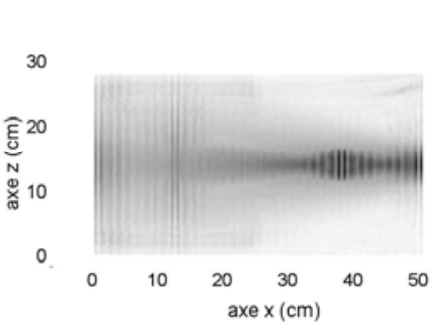

(c)
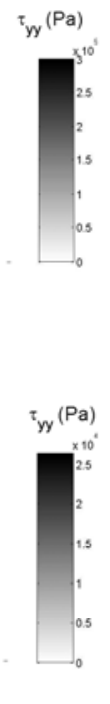

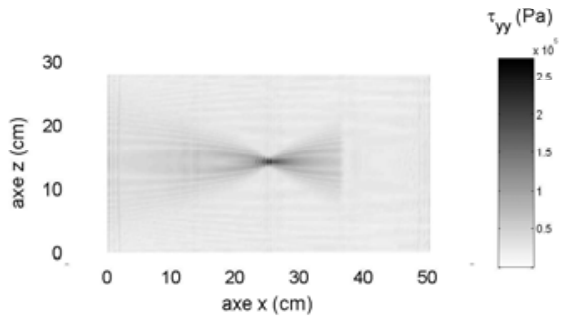

(b)

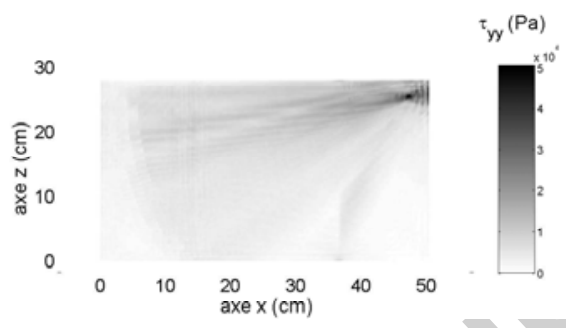

(d)

Fig. 4. Matrix of the maximum of stress amplitude along $y$ with a rebroadcasting of nonlinear signature extracted with harmonic filtering (a-c) and PI (b-d) methods and for two different defect positions (position 1: (a-b), position 2: (c-d)).

The whole results for the 2 different receiver sizes for each of the 2 defect positions are presented in Table 1, were obtained from the images of the maximum retrofocused signals. The first and second columns represent the ratio between the mean amplitude on the defect $\left(\bar{m}\left(A_{\max }\right.\right.$ (defect $\left.)\right)$ and the mean amplitude on the bulk $\left(\bar{m}\left(A_{\max }(\right.\right.$ bulk $\left.)\right)$ with PI $\left[A_{\mathrm{PI}}=\bar{m}\left(A_{\max }(\right.\right.$ defect $) / \bar{m}\left(A_{\max }\right.$ (bulk $\left.)\right]$ and harmonic filtering $\left[A_{\mathrm{Hfilt}}=\bar{m}\left(A_{\max }\right.\right.$ (defect) $) / \bar{m}\left(A_{\max }(\mathrm{bulk})\right]$, respectively. The study of the ratio $\frac{A_{P I}}{A_{H f i l t}}$, noting that if $\frac{A_{P I}}{A_{H f i l t}} \leq 1$ retrofocusing quality with harmonic filtering is better than with pulse inversion and inversely, leads to the following results. If the defect is in the middle of the bulk (i.e. between the emitter and the receivers), the retrofocusing spot is smaller when applying the harmonic filtering method (Figs. 4(a) and 4(b)). This can be explained by the dependence of the spot size on the frequency: the higher the frequency, the smaller the spot size. Using the harmonic filtering, the third harmonic is mainly returned while with the PI filtering it was mainly the second harmonic. If the defect is near an edge of the sample, the retrofocusing is only on the defect when applying the PI filtering (Figs. 4(c) and 4(d)). The energy contained in the harmonics is smaller if the defect is on the position 2 than when it is in the position 1. Moreover, when the harmonic filtering is applied, there is a small part of the fundamental frequency which is not filtered, whereas this part is canceled with PI filtering. 


\begin{tabular}{|c|c|c|l|}
\hline Defect position & $\begin{array}{c}A_{P I}=\frac{A_{\max }(\text { defect })}{A_{\max }(\text { bulk })} \\
\text { with pulse inversion }\end{array}$ & $\begin{array}{c}A_{\text {Hfilt }}=\frac{A_{\max }(\text { defect })}{A_{\max }(\text { bulk })} \\
\text { with harmonic filtering }\end{array}$ & \multicolumn{1}{|c|}{$\frac{A_{P I}}{A_{H \text { filt }}}$} \\
\hline \hline position 1 & 6,44 & 9,31 & 0,69 \\
\hline 300mm-receivers & 5,67 & 9,66 & 0,59 \\
\hline 165mm-receivers & & & 1,12 \\
\hline position 2 & 6,62 & 5,91 & 1,25 \\
\hline 300 mm-receivers & 5,63 & 4,50 & \\
\hline 165 mm-receivers & & & \\
\hline
\end{tabular}

Table 1

Comparison between the two methods of filtering for two defect positions and two source sizes.

To conclude, the harmonic filtering method is fitted for central defect, i.e. between the transmitter and the receivers, due to high level of the generated nonlinearity. On the contrary, when the generated nonlinearity is low, the pulse inversion allows to keep out all linear part of the signal. That is the reason why this last method has been chosen for the experiments.

\section{Experimental study of TR-NEWS}

Different experimental set-ups of TR-NEWS methods have been recently proposed in several papers $[13,14]$. Most of results have been obtained in complex samples which contain cracks or damaged area.

This study turns to analyse TR-NEWS performances in a simple homogeneous thick sample (aluminum) of $200 \mathrm{~mm} \times 200 \mathrm{~mm} \times 75 \mathrm{~mm}$ through the measurements of the amplitude dependence of parametric components on fundamental level.

\subsection{Experimental set-up}

Parametric interaction of acoustic waves is chosen as the nonlinear method for the extraction of the nonlinear signature. Applied as a post-treatment of TR process, it is used in order to be sure that the nonlinear signature comes from the medium and not from the transducer [32].

In order to generate this interaction, two transducers are maintained in contact on each edge of the sample. A $1 \mathrm{MHz}$ transducer (broadband) is excited by a 20 tone-burst signal at $f_{1}=490 \mathrm{kHz}$ through a $55 \mathrm{~dB}$ amplifier. A second $1 \mathrm{MHz}$ transducer (narrowband) is excited by a 35 tone-burst signal at $f_{2}=860 \mathrm{kHz}$ another $55 \mathrm{~dB}$ amplifier. In order to guarantee exact synchronisation of TR waves, all generators are triggered by an external generator. A laser vibrometer Polytec OFV-5000 is used to measure the surface velocity. The bandwidth of the decoder is about 1,5 MHz. The signal is acquired through a LeCroy 
WaveRunner 6050A oscilloscope and processed with Matlab software (Fig. 5).

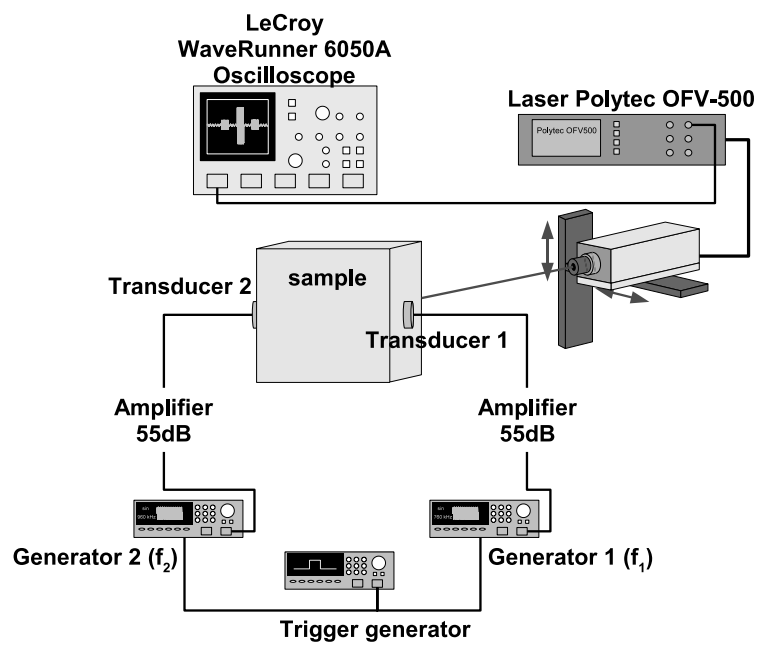

Fig. 5. TR-NEWS experimental set-up using parametric interaction.

In order to filter the components at difference $\left(f_{L}=f_{2}-f_{1}=370 \mathrm{kHz}\right)$ and sum $\left(f_{H}=f_{2}+f_{1}=1350 \mathrm{kHz}\right)$ frequencies, a PI process, presented in Sec. 2, is used. It is necessary to send two different excitations (one "positive", one "negative" (out of phase of $180^{\circ}$ )) for each frequency.

The measurement process steps for these TR-NEWS experiments with parametric interaction is done as follow:

- a 20 tone-burst signal at frequency $f_{1}=490 \mathrm{kHz}$ is emitted by the generator 1, amplified and applied to the transducer 1 ,

- signal measured by the laser vibrometer is recorded $(10 \mathrm{MHz}$ sampling rate, 45000 points and 4,5 ms duration), numerically time reversed and inverted,

- these two obtained signals $\left(f_{1+}\right.$ and $\left.f_{1-}\right)$ are stored in the internal memory of the arbitrary waveform generator 1 ,

- independently, a 35 tone-burst signal at frequency $f_{2}=860 \mathrm{kHz}$ is emitted by the generator 2 and applied to the transducer 2. Duration of emission at $f_{1}$ and $f_{2}$ are chosen identical in order to have the same electrical power,

- surface vibration measured by the laser vibrometer, on the same position as the first measurement, is recorded, numerically time reversed and inverted,

- these two obtained signals $\left(f_{2+}\right.$ and $\left.f_{2-}\right)$ are stored in emission generator 2,

- to generate parametric interaction between these two waves, signals $f_{1+}$ and $f_{2+}$ are simultaneously emitted by the two respective generators,

- on the same position of laser vibrometer measurement, the surface velocity of these two retrofocused signals is measured,

- in the same way and in order to realize pulse inversion filtering, signals $f_{1-}$ and $f_{2-}$ are also simultaneously emitted and the surface velocity is measured. 


\subsection{Study of parametric interaction}

Figure 6 presents the retrofocused signals, obtained after the process detailed previously. These signals are studied in term of nonlinear response and typically at $f_{L}$ and $f_{H}$ frequencies [33].

\subsubsection{Experimental retrofocused signals}

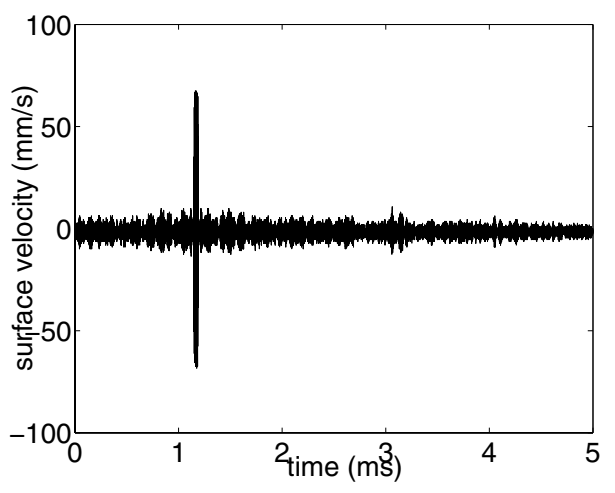

(a)

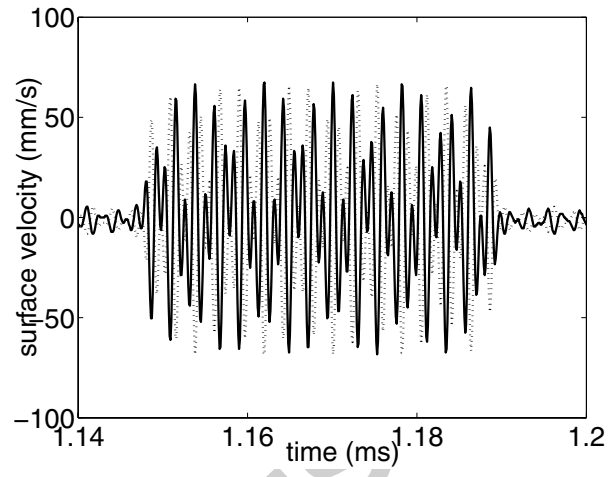

(b)

Fig. 6. Positive (solid line) and negative (dashed line) retrofocused signals (a) for simultaneous emission at frequencies $f_{1}=490 \mathrm{kHz}$ and $f_{2}=860 \mathrm{kHz}$ and (b) between $t=1.14 \mathrm{~ms}$ and $t=1.2 \mathrm{~ms}$.

The study of the retrofocused signals is realized in the area defined between $t=1,14 \mathrm{~ms}$ and $t=1,2 \mathrm{~ms}$. In this area, a spectral investigation of the sum of the two phase inverted signals is done in order to evaluate the level of signals at $f_{L}$ and $f_{H}$ frequencies (Fig. 7). In spite of pulse inversion filtering, a small level of fundamental frequency at $f_{1}=490 \mathrm{kHz}$ and $f_{2}=860 \mathrm{kHz}$ persists after PI filtering on this spectral representation. The remaining line at fundamental frequency probably comes from the ADC converter and/or the intrinsic triggering jitter. Retrofocused signals at each frequency are represented and it is possible to notice that signals at $f_{L}$ and $f_{H}$ frequencies are present. 


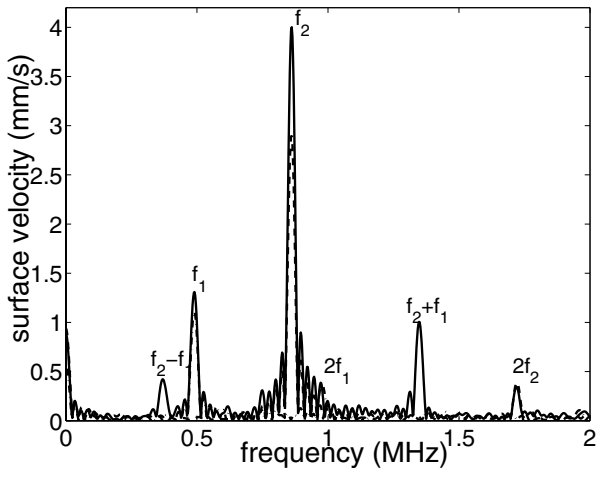

(a)

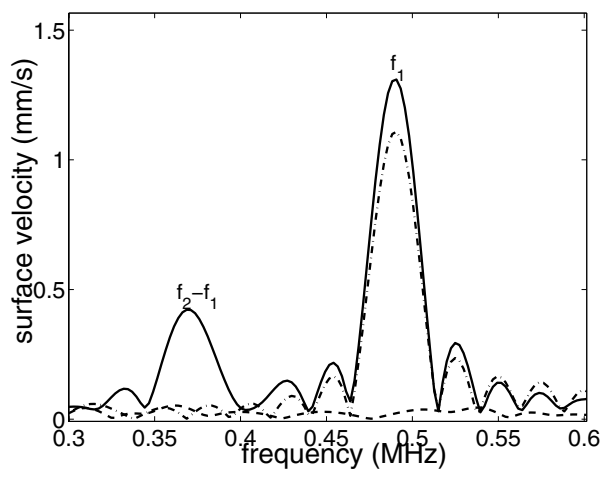

(b)

Fig. 7. Spectrum of the sum of retrofocused signals (in-phase and out-of-phase) (a) between $0 \mathrm{MHz}$ and $2 \mathrm{MHz}$ and (b) around the difference frequency : $f_{1}$ emitted alone (dash-dotted line), $f_{2}$ emitted alone (dashed line) and $f_{1}$ and $f_{2}$ emitted together (solid line).

In the case of emission at a single frequency, as it is expected in nonlinear propagation, we notice apparition of second harmonic $\left(2 f_{1}=980 \mathrm{kHz}\right.$ and $2 f_{2}=1.72 \mathrm{MHz}$ ). The problem is to know if this nonlinearity is produced by the wave propagation in the medium or directly by the transducer and the electrical emission channel [32]. This is the reason why parametric interaction seems to be interesting to avoid this problem .

\subsubsection{Amplitude dependence of fundamental level}

3.2.2.1 Parametric interaction equations Nonlinear acoustic wave equation first derived by Westervelt [34] takes into account physical nonlinearity in the equation of state and geometrical nonlinearity:

$$
\Delta p-\frac{1}{c_{0}^{2}} \frac{\partial^{2} p}{\partial t^{2}}=-\frac{\beta}{\rho_{0} c_{0}^{4}} \frac{\partial^{2} p^{2}}{\partial t^{2}}
$$

where $p$ is the pressure, $c_{0}$ the celerity, $\rho_{0}$ the volumetric mass density and $\beta$ the nonlinear parameter. For interaction of two frequency waves $\left(\omega_{1}\right.$ and $\left.\omega_{2}\right)$, the source densities for the difference $f_{L}$ and sum $f_{H}$ frequencies are :

$$
\begin{aligned}
q_{L} & =\frac{\beta L\left(\omega_{1}-\omega_{2}\right)^{2}}{2 \rho_{0}^{2} c_{0}^{4}} P_{1} P_{2}, \\
q_{H} & =\frac{\beta L\left(\omega_{1}+\omega_{2}\right)^{2}}{2 \rho_{0}^{2} c_{0}^{4}} P_{1} P_{2},
\end{aligned}
$$

where $P_{1,2}$ is the amplitude of the fundamental frequency wave $f_{1,2}$ and $L$ is the propagation length. 
In order to investigate the nonlinear signature on the focus spot, the dependence of the parametric interaction components $q_{L}$ and $q_{H}$ versus amplitude of the fundamental components $P_{1}$ and $P_{2}$ at frequencies $f_{1}=490 \mathrm{kHz}$ and $f_{2}=860 \mathrm{kHz}$ is achieved.

3.2.2.2 Experimental results TR-NEWS experiments were realized in a sample of aluminum $\left(200 \times 200 \times 75 \mathrm{~mm}^{3}\right)$ with a simultaneous variation of the amplitude of the generator of each wave $\left(f_{1}=490 \mathrm{kHz}\right.$ and $\left.f_{2}=860 \mathrm{kHz}\right)$ between $150 \mathrm{mV}$ and $400 \mathrm{mV}$ before amplification. To study the parametric interaction level versus these amplitudes, the slope of the amplitudes of parametric components versus the product of the amplitude of the fundamental frequency components is given in Fig. 8 in logarithm scale. Three different experiments have been conducted in order to test the repeatability.

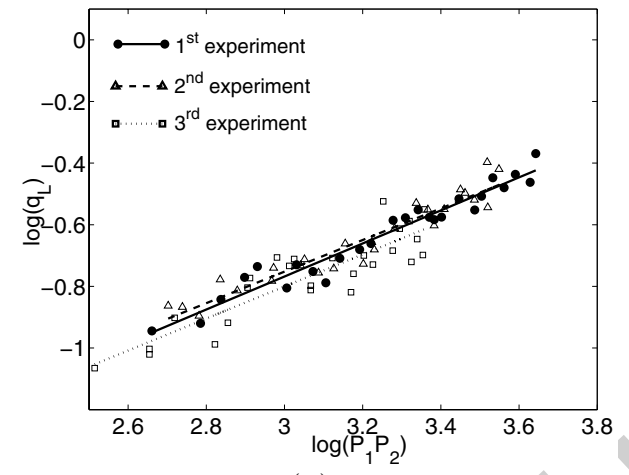

(a)

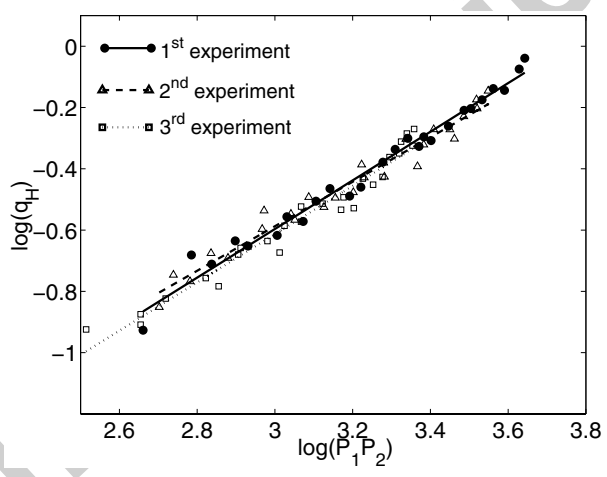

(b)

Fig. 8. Evolution of the amplitude of (a) the difference-frequency component $q_{L}$ and (b) the sum-frequency component $q_{H}$ versus the product of the amplitude of the fundamental frequency components for three consecutive experiments.

In Table 2, the linear approximations of the evolution of the parametric components versus the product of the amplitude of fundamental components are presented.

The theoretical evolution of the logarithm of $q_{L}$, defined in eq. (5), versus the logarithm of $P_{1} P_{2}$ is

$$
\log \left(q_{L}\right)=\log \left(P_{1} P_{2}\right)+\log \left(\frac{\beta L\left(\omega_{1}-\omega_{2}\right)^{2}}{2 \rho_{0}^{2} c_{0}^{4}}\right) .
$$

Despite a good repeatability of measurements ( $5 \%$ for the difference frequency and $7 \%$ for the sum frequency), the expected expressions (Eq. (7)) are not experimentally verified. 


\begin{tabular}{|c||c|}
\hline & Linear approximations for the difference-frequency \\
\hline \hline $1^{\text {st }}$ experiment & $\log \left(q_{L}\right)=0,54 \log \left(P_{1} P_{2}\right)-2,38$ \\
\hline $2^{\text {nd }}$ experiment & $\log \left(q_{L}\right)=0,51 \log \left(P_{1} P_{2}\right)-2,29$ \\
\hline $3^{\text {rd }}$ experiment & $\log \left(q_{L}\right)=0,52 \log \left(P_{1} P_{2}\right)-2,36$ \\
\hline & Linear approximations for the sum-frequency \\
\hline \hline $1^{\text {st }}$ experiment & $\log \left(q_{H}\right)=0,79 \log \left(P_{1} P_{2}\right)-2,98$ \\
\hline $2^{\text {nd }}$ experiment & $\log \left(q_{H}\right)=0,73 \log \left(P_{1} P_{2}\right)-2,77$ \\
\hline $3^{\text {rd }}$ experiment & $\log \left(q_{H}\right)=0,79 \log \left(P_{1} P_{2}\right)-2,97$ \\
\hline
\end{tabular}

Table 2

Polynomial approximations for the difference and the sum frequencies on aluminum sample for three consecutive experiments.

Throughout this study, the coefficient of the polynomial approximation has never reached the expected value $(=1)$. The theory of parametric interaction, developed by Wertervelt, takes into account only the case of two collinear waves. In our case, the generated waves are retrofocused on a point; consequently, the hypothesis of collinearity seems to be not verified. Nevertheless, a nonlinear signature can be observed on the retrofocusing area. The damaged zone influence on these signals could be studied with the TR-NEWS method.

\section{Conclusion}

Feasibility to realize defect imaging in a bulk using nonlinear signatures extracted from NEWS combined with TR methodology, has been demonstrated by means of $2 \mathrm{D}$ simulations. The outcome of the simulations firstly reinforces our belief in the usefulness of the NEWS-TR method as a tool for microdamage imaging. Secondly the experimental study about parametric interaction shows that it is possible to observe a nonlinear signature using the TR-NEWS method. Moreover it seems to be very interesting to develop this method combining nonlinear treatment like parametric interaction in order to minimize the nonlinearity due to the measurement equipment, with TR process, doing a space and time retrofocusing.

\section{Acknowledgements}

This work has been supported by the European Community Sixth Framework Programme AERONEWS: Specific Targeted Research: FP6-502927. 


\section{References}

[1] K.E. Van Den Abeele, A. Sutin, J. Carmeliet and P. Johnson, Micro-damage diagnostics using nonlinear elastic wave spectroscopy (NEWS), NDT \& $E$ International 34, 239-248 (2001)

[2] V. Nazarov and A. Sutin, Harmonic generation in the propagation of elastic waves in nonlinear solid media, Sov. Phys. Acoust. 35, 410-413 (1989)

[3] A. Moussatov, B. Castagnde and V. Gusev, Frequency up-conversion and frequency down-conversion of acoustic waves in damaged materials, Phys. Lett. A 301, 281-290 (2002)

[4] K.E. Van Den Abeele, P. Johnson and A. Sutin, Nonlinear elastic wave spectroscopy (NEWS) techniques to discern material damage I. Nonlinear wave modulation spectroscopy (NWMS), Res. Nondestruct. Eval. 12, 17-30 (2000)

[5] M. Vila, F. Vander Meulen, S. Dos Santos, L. Haumesser and O. Bou Matar, Contact phase modulation method for acoustic nonlinear parameter measurement in solid, Ultrasonics 42, 1061-1065 (2004)

[6] K.E. Van Den Abeele, K. Van de Velde and J. Carmeliet, Inferring the degradation of pultruded composites composites from dynamic nonlinear resonance measurements, Polym. Compos. 22, 555-567 (2001)

[7] M. Ben Tahar, M. Griffa, H. Elaqra, R. El Guerjouma and M. Scalerandi, Hysteretic elasticity in damaged concrete: quantitative analysis of slow and fast dynamics, Phys. Rev. B 73, 014116 (2006)

[8] R. Guyer and P. Johnson, Nonlinear mesoscopic elasticity: evidence for a new class of materials, Phys. Today, 30-36 (1999)

[9] K. McCall and R. Guyer, Equation of state and wave propagation in hysteretic nonlinear elastic materials, Journal of Geophysical research 99 , 23887-23897 (1994).

[10] M. Fink, Time Reversal of Ultrasonic Fields, Part I, II and III, IEEE Trans. Ultrason. Ferroelectr. Freq. Control 39, 555-592 (1992)

[11] M. Fink, Time reversed acoustics, Phys. Today 50, 34-40 (1997)

[12] A. Sutin, J.A. TenCate and P.A. Johnson, Development of nonlinear time reversed acoustics (NLTRA) for applications to crack detection in solids, in Proc. of the 5th World Congress on Ultrasonics, 121-124 (2003)

[13] T.J. Ulrich, P.A. Johnson and A. Sutin, Imaging nonlinear scatterers applying the time reversal mirror, J. Acoust. Soc. Am., 119, 1514-1518 (2006)

[14] P.-Y. Le Bas, K. Van Den Abeele, S. Dos Santos, T. Goursolle and O. Bou Matar, Experimental analysis for nonlinear Time Reversal imaging of damaged materials, in Proc. of the 9th European Conference on Non-Destructive Testing (2006)

[15] O. Bou Matar, S. Dos Santos, J. Fortineau, T. Goursolle, L. Haumesser and F. Vander Meulen, Pseudo spectral simulations of elastic waves propagation in heterogeneous nonlinear hysteretic medium, in Proc. of the 17th International Symposium of Nonlinear Acoustics (2005) 
[16] T. Goursolle, S. Callé, S. Dos Santos and O. Bou Matar, A two-dimensional pseudospectral model for time reversal and nonlinear elastic wave spectroscopy, J. Acoust. Soc. Am., 122, 3220-3229 (2007)

[17] A.S. Gliozzi, M. Griffa and M. Scalerandi, Efficiency of time-reversed acoustics for nonlinear damage detection in solids, J. Acoust. Soc. Am., 120(5), 2506-2517 (2006)

[18] O. Bou Matar, S. Dos Santos, S. Call, T. Goursolle, S. Vanaverbeke and K. Van Den Abeele, Simulations of nonlinear Time Reversal imaging of damaged materials, in Proc. of the 9th European Conference on Non-Destructive Testing (2006)

[19] G. Zumpano and M. Meo, A new nonlinear elastic time reversal acoustic method for the identification and localisation of stress corrosion cracking in welded platelike structures - A simulation study, Int. J. Solids Struct., 44, 3666-3684 (2007)

[20] T.J. Ulrich, P.A. Johnson and R. Guyer, Interaction dynamics of elastic waves with a complex nonlinear scatterer through the use of a time reversal mirror, Phys. Rev. Lett., 98, 104301 (2007)

[21] I. Mayergoysz, Hysteresis models from the mathematical and control theory points of view, J. Appl. Phys. 57, 3803-3805 (1985)

[22] R. Guyer, K. McCall and G. Boitnott, Hysteresis, discrete memory and nonlinear wave propagation in rock : a new paradigm, Phys. Rev. Letters 74, 3491-3494 (1995)

[23] K. Van Den Abeele, F. Schubert, V. Aleshin, F. Windels and J. Carmeliet, Resonant bar simulations in media with localized damage, Ultrasonics 42, 1017$1024(2004)$

[24] M. Scalerandi, V. Agostini, P.P. Delsanto, K. Van Den Abeele and P. Johnson, Local Interaction Simulation Approach to modelling nonclassical nonlinear elastic behavior in solids, J. Acoust. Soc. Am. 113, 3049-3059 (2003)

[25] J. Dellinger, D. Vasicek and C. Sondergeld, Kelvin notation for stabilizing elastic-constant inversion, Rev. Inst. Fr. Pet 53, 709-719 (1998)

[26] O. Bou Matar, V. Preobrazhensky and P. Pernod, Two-dimensionnal axisymmetric numerical simulation of supercritical phase conjugation of ultrasound in active solid media, J. Acoust. Soc. Am. 118, 2880-2890 (2005)

[27] B. Fornberg and D.M. Sloan, A review of pseudospectral methods for solving partial differential equations, Acta Numerica, 203-267 (1994)

[28] Q.H. Liu, The PSTD algorithm: A time-domain method requiring only two cells per wavelength, Microwave Opt. Tech. Lett. 15, 158-165 (1997)

[29] B. Fornberg, The pseudospectral method : Comparisons with finite differences for the elastic wave equation, Geophysics 52, 483-501 (1987)

[30] M. Ghrist, B. Fornberg and T. A. Driscoll, Staggered time integrators for wave equations, SIAM J. Numer. Anal. 38, 718741 (2000)

[31] D.H. Simpson, C.T. Chin and P.N. Burns, Pulse inversion Doppler: a new method for detecting nonlinear echoes from microbubble contrast agents, IEEE Trans. Ultrason. Ferroelectr. Freq. Control 46, 372-382 (1999) 
[32] J. Fortineau, F. Vander Meulen, T. Goursolle and L. Haumesser, Experimental study of the nonlinearity from ultrasonic transducers, IEEE Ultrason. Symp. 39, 1786-1789 (2006)

[33] T. Goursolle, J. Fortineau, S. Callé and S. Dos Santos, Localized ultrasonic parametric interaction near cracked area in thin samples with nonlinear elastic wave spectroscopy-based time reversal, In Proceedings of International Congress of Ultrasonics, Vienna (2007)

[34] P.J. Westervelt, Parametric acoustic array, J. Acoust. Soc. Am. 35, 535-537 (1963) 